\title{
Effectiveness of Respectful Maternity Care Interventions for Women Using Maternal Health Care: A Systematic Review Protocol
}

Habtamu Kasaye ( $\square$ habtekebe@gmail.com )

Wollega University https://orcid.org/0000-0003-3759-2604

Kathleen Baird

University of Technology Sydney Faculty of Health

Annabel Sheehy

University of Technology Sydney Faculty of Health

Vanessa Scarf

University of Technology Sydney Faculty of Health

Allison Cummins

The University of Newcastle

\section{Protocol}

Keywords: maternal health, systematic review, Critical Appraisal Skills Program, GRADE criteria

Posted Date: December 21st, 2021

DOI: https://doi.org/10.21203/rs.3.rs-1159194/v1

License: (c) (1) This work is licensed under a Creative Commons Attribution 4.0 International License. Read Full License 


\section{Abstract}

Background: Studies have indicated the mistreatment of women during maternal health care provision as being a driving factor for women eschewing professional care. The mistreatment of women is not only a violation of human rights, but also a significant contributor to poor-quality maternal care. Various strategies aimed at preventing the mistreatment of women and enhancing respectful maternity have been proposed, however, the positive effects and outcomes of these interventions necessitate further clarity. This systematic review will examine the effectiveness of respectful maternity care intervention programs in preventing the mistreatment of women whilst enhancing respectful maternity care in health facilities.

Methods: A systematic review will be undertaken according to the Preferred Reporting Items for Systematic and Meta-Analysis (PRISMA)2020. Both published and unpublished randomised, nonrandomised controlled and observational studies obtained from PubMed, CINHAL, EMBASE, AJOL, and other databases and grey literature sources will be assessed against an inclusion and exclusion criteria to include in the review. Two independent reviewers will assess the papers selected for retrieval to ensure methodological validity. Standardised critical appraisal instruments from the Critical Appraisal Skills Program (CASP) and Joanna Briggs Institute Meta-Analysis of Statistics Assessment and Review Instrument (JBI-MAStARI) will be used. The data will be extracted from papers included in the review using two independent reviewers' standardised data extraction tool. Evidence synthesis and, wherever possible, meta-analysis will be performed. Certainty of the evidence will be assessed by using GRADE criteria.

\section{Systematic review protocol registration: Submitted to PROSPERO on $9^{\text {th }}$ of November 2021 (ID. 287049).}

\section{Introduction}

Continuing the international pursuit of progress as set out by the Millennium Development Goals (MDGs), the United Nations reaffirmed the objectives with the introduction of the Sustainable Development Goals (SDGs) in 2016 [1]. The global objective of these targets was to promote peace, well-being and health for all and the eradication of poverty [1]. The World Health Organization (WHO) was one amongst many international organisations committed to achieving the Development Goals. With the purpose to specifically address maternal health SDG targets, the WHO directed attention to end preventable maternal deaths and boost greater accessibility to sexual and reproductive care services [2]. These all-important priorities, disclosed in the Global strategy for women's, children's and adolescents' health, 2016-2030, have the purpose of accelerating the then 2.9 per cent annual reduction rate of the global Maternal Mortality Ratio (MMR) from 210 per 100,000 live births in 2015, to less than 70 per 100,000 live births $2030[2,3]$. As is the case with other economic, poverty and social indicators, maternal mortality is not equally distributed across the globe, with nine out of every ten of the annual 295,000 maternal deaths occurring in low-income and middle-income countries [4]. 
Quality, skilled and accessible maternal health care before, during and after childbirth are vital necessities that can address the global disparities of maternal mortality and morbidity and mitigate the circumstances leading to maternal death $[5,6]$. Following the implementation of targets to reduce maternal mortality and the sustained efforts to enhance the accessibility of maternal health services, skilled birth attendance across the globe increased from 59-83\% in the years 1990 to 2020 [7]. These gains, however, exposed health system and facilities' failures, whereby women were not seeking skilled birth attendance despite availability of attendants. Studies have indicated the mistreatment of women during maternal health care provision as a driving factor for women eschewing professional care [8-12]. Women who have been mistreated by health care providers lose trust in these professionals and are therefore less likely to seek care. Bypassing professional maternity care hinders strategies aimed to reduce maternal deaths across the globe $[13,14]$.

In response to this critical issue, the White Ribbon Alliance established a respectful maternity care charter in 2011 [15]. The WHO also developed a statement for the prevention and elimination of the mistreatment of women during maternity care [16], which was succeeded by the Respectful Maternity Care (RMC) recommendation as being a critical component of routine quality care of women and their children [17]. Included therein is a mandate that the protection of a woman's privacy, confidentiality, and dignity should be standardised in all maternity care practices and advocates for continuous support of women being a routine aspect of maternity care in order to be a safeguard against harm and abuse [18].

Various strategies aimed at the prevention of mistreatment of women have been proposed. These include strategies for improving access to midwifery-led care [19], the continued commitment to community mobilisation and self-advocacy to call for safe, respectful maternity care, and approaches to amend attitudes, values and behaviours of health care providers which contribute to poor quality and unsafe care of women $[20,21]$. It is currently unclear, however, whether these strategies have been effective in enhancing RMC and preventing mistreatment [18]. Reviewing the RMC policies for their potential to reduce occurrences of disrespect and abuse, Downe et al. [22] reported that multi-component RMC policies could accomplish change. Obtaining further clarity about which interventions have achieved positive changes for safe maternity care is now required. This systematic review will address this gap, identifying the types of RMC interventions that are used to prevent mistreatment, as well as their effectiveness in avoiding mistreatment and enhancing safe and respectful maternity care in health facilities.

Study aim

The primary aim of this systematic review is to examine the available evidence on interventions that aim to enhance respectful maternity care experiences and to identify the types of interventions used, as well as their effect in preventing mistreatment of women during maternity care. It will also report on the implementation and effectiveness of respectful maternity care programs through examining their reported outcomes. The proposed systematic review will answer the following research questions: 
1. What maternity care intervention programs have been implemented across low-and-middle income countries aiming to prevent mistreatment of women?

2. Were respectful maternity care intervention programs effective in preventing mistreatment of women during maternity care?

3. Were maternity care interventions effective in enhancing women's respectful maternity care experiences?

\section{Methods}

\section{Study design}

A systematic review will be undertaken to critically assess and evaluate available research studies that address the interventions and strategies implemented to eliminate the mistreatment of women in maternity care and facilitate respectful maternity care.

\section{Review method}

Studies that have evaluated the effects of health interventions to prevent mistreatment and enhance women's safe and respectful maternity care will be reviewed according to the Preferred Reporting Items for Systematic and Meta-Analysis-2020 (PRISMA-2020) guideline [23]. The findings of the selected research studies will be described and evaluated, and a meta-analysis will be undertaken. The overall aim is the critical evaluation of the state of knowledge on respectful maternity care health practices.

\section{Criteria for selecting studies for this review}

Studies will be selected according to the PICOSS (participants/ population, intervention, comparisons, outcomes, study designs, and settings) by following the following inclusion and exclusion criteria.

\section{Inclusion criteria}

Studies in English at any date will be included when:

- The intervention was designed to prevent or decrease mistreatment of women during maternity care (physical abuse, verbal abuse, sexual abuse, privacy, non-consented and non-confidential care, stigma/discrimination, abandonment, detention)

- Interventions were intended to enhance respectful maternity care

- The intervention was designed to impact the experiences of women during maternity care in health facilities 
- The research has been conducted in randomised controlled trials, observational longitudinal pre- and post-studies

\section{Exclusion criteria}

Studies will be excluded when:

- They are qualitative investigations, book chapters, case reports, letters, opinions, and editorials

- A study does not report to the comparison of outcome rates between interventional and comparator groups

- Studies were not designed primarily to prevent mistreatment of women or respectful care enhancement, but were instead intended to increase service utilisation and equity

- The intervention is primarily designed to decrease specific intrapartum interventions

- Articles are found to be fraudulent or may for other reasons have been retracted since publication will be excluded from systematic review and meta-analysis.

\section{Population}

The review will consider studies that have investigated the effectiveness of interventions that were applied across service and/or a community, health service providers and/or a health facility for the prevention of mistreatment of women and ensure respectful maternity care for all women in low-and middle-income countries during maternity care (during pregnancy, birth, and postnatal care).

\section{Interventions}

The review will consider studies that evaluated the effectiveness of RMC interventions aimed at minimising disrespect and abuse in maternity care. Interventions that aimed to reinforce the validity of respectful maternity care may include studies regarding facility-based quality improvement processes, or studies reporting on the effectiveness of interventions. Examples of interventions could be those that, adhered to RMC guidelines and policies, provided RMC training, ensured accessible essential equipment, medicines and health supplies, and when facilities supported linkages between the facility and the community.

\section{Comparators}


The effectiveness of RMC interventions will be compared to usual maternity care provided in health facilities in the absence of any interventions at an individual, health facility or community level related to improving respectful care or preventing disrespectful and abusive care.

\section{Outcomes}

The primary outcomes will be mistreatment of women (observed by investigators or self-reported by women). It is important to note that the mistreatment of women has various interpretations across the world because the standard of health care and the way that health care is organised and experienced differs around the globe. For the purpose of this systematic review mistreatment will include the explicit experiences of verbal abuse, physical abuse, neglect, stigma and discrimination, poor communication or other forms of mistreatment related to health care providers or health care facilities, as described in comprehensive global systematic review conducted by Bohren and et al. [24].

The next potential outcome will be the provision of respectful maternity care (RMC). Respectful maternity care has been provided when health care providers deliver maternity care which is underpinned by the ethical principles of non-maleficence and beneficence. Using findings from systematic reviews, Shakibazadeh et al. [25] developed twelve domains of RMC to clarify what constitutes respectful care. These twelve domains will be used as guiding measures of respectful maternity care.

\section{Types of studies going to be included in review}

Randomised, non-randomised controlled and observational studies identifying the effectiveness of respectful maternity care interventions focusing on the prevention of the mistreatment of women or enhancing respectful maternity care will be included in the review.

\section{Search methods for identification of studies}

The search strategy aims to retrieve both published and unpublished studies. A three-step search strategy will be utilised. An initial search of PubMed and CINAHL will be undertaken, followed by an analysis of the text and words contained within the journal title and abstract, and the index terms used to describe the article. A second search using all identified keywords and index terms will then be undertaken across a variety of databases and other sources, including EBSCO Nursing/Academic Edition, African Index Medicus, African Journals Online (AJOL), Scopus and Google Scholar. Thirdly, a manual search of the reference lists of all identified papers and reports will done to locate any additional studies citied within the identified papers.

Only studies written and published in English will be considered for inclusion. A search for unpublished studies will also include ProQuest's dissertation and thesis database, grey literature from search engines such as Google, and finally those of various universities databases. Keywords will include 'mistreatment', 
'disrespect and abuse', 'maternity care', 'antenatal care', 'birth', 'postnatal care', 'interventions', 'programs', and 'policy'. The details of PubMed search strategies are given in a supplementary file (S1).

\section{Study selection}

The results from all bibliographic searches will be exported into Endnote version X9 [26], which will be used to manage and store relevant studies. Citations will be exported to Covidence systematic review software (Veritas Health Innovation, Melbourne, Australia. Available at www.covidence.org). Duplicates of identified studies will then be removed in Covidence. Both software packages used are licensed by the University of Technology Sydney.

The title and abstract of all remaining studies will be assessed for their relevance based on inclusion and exclusion criteria by HK and VS. Those articles considered potentially relevant to the aims of this systematic review will have their abstract read to assess for the utility of full-text screening. Therefore, any study or report noted to be relevant will be read in full and determined whether suitable for the final selection. The screening process as specified by the Studies screening PRISMA 2020 flow diagram [23] will be used. This diagram is presented in figure 1.

\section{Data collection and analysis}

\section{Assessment of methodological quality}

Papers selected for retrieval will be assessed independently by two reviewers to ensure methodological validity. The standardised critical appraisal instruments from the Critical Appraisal Skills Program (CASP) and Joanna Briggs Institute Meta-Analysis of Statistics Assessment and Review Instrument (JBIMAStARI) will be used. When a difference of opinion occurs between the two reviewers, the issue will be resolved through them discussing the specific study, and a consensus will be reached with the involvement of a third reviewer, AS.

\section{Data collection}

Data will be extracted from papers using a standardised data extraction tool from JBI-MAStARI by two independent reviewers within the research team. The data extracted will include specific details about the study population, interventions used, study methods and outcomes of significance in relation to the review question and objectives.

\section{Data synthesis}


The papers will be pooled in a statistical meta-analysis using Stata version 15 meta-analysis. All results will be subject to double data entry. When reviewing RMC interventions that have been aimed at preventing mistreatment of women or improving experiences of RMC, different categories of interventions will be combined in a single meta-analysis to address the question: 'what is the effect of RMC interventions on reducing or preventing mistreatment of women?'.

The effect sizes will be expressed as odds ratio for categorical data (mistreatment among interventional groups compared to control/usual care groups) and their $95 \%$ confidence intervals will be calculated for analysis. Heterogeneity will be assessed statistically using the standard Chi-square and I-squared and explored using subgroup analyses based on the study designs, types of RMC interventions (community intervention, health system intervention, health facility or individual health care providers targeted interventions). If the results of sub-group analysis indicated considerable heterogeneity via an I-squared of greater than $20 \%$, the random effect model will then be reported. Where statistical pooling of variables is not possible the findings will then be presented in description form, including the use of tables and figures to aid in data presentation where appropriate.

\section{Assessing certainty in the findings}

The Grading of Recommendations, Assessment, Development and Evaluation (GRADE) approach for grading the certainty of evidence and a Summary of Findings will be created using GRADEPro.

\section{Discussion}

This systematic review will identify the best available evidence regarding the effectiveness of respectful maternity care interventions with the purpose of providing useful information for strategy development for the minimisation of mistreatment of women during maternity care within health facilities. In addition, it will also identify whether the implementation of an intervention improved respectful maternity care culture within the health care facility it was established in. Specific to the homeland of the first author, almost three out of four women in a study in Western Ethiopia reported experiencing at least one form of disrespect and abuse in their maternity care [27]. This gross mistreatment of the women in this locale has the potential to influence future decisions about maternity care in any subsequent pregnancy and childbirth. Women's accounts of negative experiences circulating in the community could also influence others not to utilise health facilities for their own health needs. The evidence generated from this review can be used by those who design, develop, and implement interventions aimed at minimising the mistreatment women within maternity care health facilities. Furthermore, we anticipate that the findings of this review will be a useful to a variety of stakeholders who wish to implement a model of maternity care where women and their families are only treated with respect and kindness.

\section{Abbreviations}

CASP: Critical Appraisal Skills Program 
GRADE: Grading of recommendations, assessment, development, and evaluations

JBI-MAStARI: Joanna Briggs Institute Meta-Analysis of Statistics Assessment and Review Instrument MDGs: Millennium Development Goals

MMR: Maternal Mortality Ratio

PRISMA-P: Preferred Reporting Items for Systematic Review and Meta-Analysis extension for protocols

PRISMA-2020: Preferred Reporting Items for Systematic and Meta-analysis-2020

RMC: Respectful Maternity Care

SDGs: Sustainable Development Goals

WHO: World Health Organization

\section{Declarations}

\section{Ethics approval and consent to participate}

Not applicable

\section{Consent for publication}

Not applicable

\section{Availability of data and materials}

Not applicable

\section{Competing interest}

The authors declare that they have no competing interests.

\section{References}

1. United Nations. Transforming our world: the 2030 Agenda for Sustainable Development. Division for Sustainable Development Goals: New York, NY, USA2015.

2. World Health Organization. Strategies towards ending preventable maternal mortality (EPMM). 2015.

3. World Health Organization. Global strategy for women's, children's and adolescent's health (20162030). Geneva: WHO2015.

4. World Health Organization. Trends in maternal mortality 2000 to 2017: estimates by WHO, UNICEF, UNFPA, World Bank Group and the United Nations Population Division. Geneva: World Health 
Organization; 20192019.

5. Downe S, Finlayson K, Oladapo OT, Bonet M, Gülmezoglu AM. What matters to women during childbirth: A systematic qualitative review. PLoS One. 2018;13(4):e0194906.

6. Alkema L, Chou D, Hogan D, Zhang S, Moller AB, Gemmill A, et al. Global, regional, and national levels and trends in maternal mortality between 1990 and 2015, with scenario-based projections to 2030: a systematic analysis by the UN Maternal Mortality Estimation Inter-Agency Group. Lancet. 2016;387(10017):462-74.

7. United Nations. The Sustainable Development Goals Report 2021. 2021.

8. Jha P, Christensson K, Svanberg AS, Larsson M, Sharma B, Johansson E. Cashless childbirth, but at a cost: A grounded theory study on quality of intrapartum care in public health facilities in India. Midwifery. 2016;39:78-86.

9. Bohren MA, Mehrtash H, Fawole B, Maung TM, Balde MD, Maya E, et al. How women are treated during facility-based childbirth in four countries: a cross-sectional study with labour observations and community-based surveys. Lancet. 2019;394(10210):1750-63.

10. Bohren MA, Hunter EC, Munthe-Kaas HM, Souza JP, Vogel JP, Gülmezoglu AM. Facilitators and barriers to facility-based delivery in low- and middle-income countries: a qualitative evidence synthesis. Reprod Health. 2014;11(1):71.

11. Minckas N, Gram L, Smith C, Mannell J. Disrespect and abuse as a predictor of postnatal care utilisation and maternal-newborn well-being: a mixed-methods systematic review. BMJ Glob Health. 2021;6(4):e004698.

12. Finlayson $\mathrm{K}$, Downe $\mathrm{S}$. Why do women not use antenatal services in low-and middle-income countries? A meta-synthesis of qualitative studies. PLoS medicine. 2013;10(1):e1001373.

13. Maung TM, Mon NO, Mehrtash H, Bonsaffoh KA, Vogel JP, Aderoba AK, et al. Women's experiences of mistreatment during childbirth and their satisfaction with care: findings from a multicountry community-based study in four countries. BMJ Glob Health. 2021;5(Suppl 2).

14. Jungari S, Sharma B, Wagh D. Beyond Maternal Mortality: A Systematic Review of Evidences on Mistreatment and Disrespect During Childbirth in Health Facilities in India. Trauma Violence Abuse. 2019:1524838019881719.

15. Respectful Maternity Care: The Universal rights of women and newborns, (2011).

16. World Health Organization. The prevention and elimination of disrespect and abuse during facilitybased childbirth: WHO statement. World Health Organization; 2015.

17. World Health Organization. Quality, equity, dignity: the network to improve quality of care for maternal, newborn and child health: strategic objectives. 2018.

18. World Health Organization. WHO recommendations on intrapartum care for a positive childbirth experience: World Health Organization; 2018.

19. Binfa L, Pantoja L, Ortiz J, Gurovich M, Cavada G, Foster J. Assessment of the implementation of the model of integrated and humanised midwifery health services in Chile. Midwifery. 2016;35:53-61. 
20. Kujawski SA, Freedman LP, Ramsey K, Mbaruku G, Mbuyita S, Moyo W, et al. Community and health system intervention to reduce disrespect and abuse during childbirth in Tanga Region, Tanzania: A comparative before-and-after study. PLoS Med. 2017;14(7):e1002341.

21. Abuya T, Ndwiga C, Ritter J, Kanya L, Bellows B, Binkin N, et al. The effect of a multi-component intervention on disrespect and abuse during childbirth in Kenya. BMC Pregnancy Childbirth. 2015;15(1):224.

22. Downe S, Lawrie TA, Finlayson K, Oladapo OT. Effectiveness of respectful care policies for women using routine intrapartum services: a systematic review. Reprod Health. 2018;15(1):23.

23. Page MJ, McKenzie JE, Bossuyt PM, Boutron I, Hoffmann TC, Mulrow CD, et al. The PRISMA 2020 statement: an updated guideline for reporting systematic reviews. Bmj. 2021;372:n71.

24. Bohren MA, Vogel JP, Hunter EC, Lutsiv O, Makh SK, Souza JP, et al. The Mistreatment of Women during Childbirth in Health Facilities Globally: A Mixed-Methods Systematic Review. PLoS Med. 2015;12(6):e1001847; discussion e.

25. Shakibazadeh E, Namadian M, Bohren MA, Vogel JP, Rashidian A, Nogueira Pileggi V, et al.

Respectful care during childbirth in health facilities globally: a qualitative evidence synthesis. BJOG. 2018;125(8):932-42.

26. The EndNote Team. EndNote. EndNote X9 ed. Philadelphia, PA: Clarivate; 2013.

27. Bobo FT, Kasaye HK, Etana B, Woldie M, Feyissa TR. Disrespect and abuse during childbirth in Western Ethiopia: Should women continue to tolerate? PLoS ONE. 2019;14(6).

\section{Figures}


Identification of studies via databases and registers

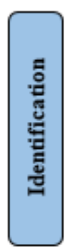

Records identified from*:

PubMed ( $\mathrm{n}=$ )

CINHAL ( $\mathrm{n}=$ )

Ovid Embase $(\mathrm{n}=)$

Registers $(\mathrm{n}=$ )

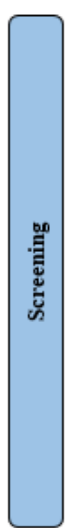

ㅁّㅁ

reasons $(\mathrm{n}=)$
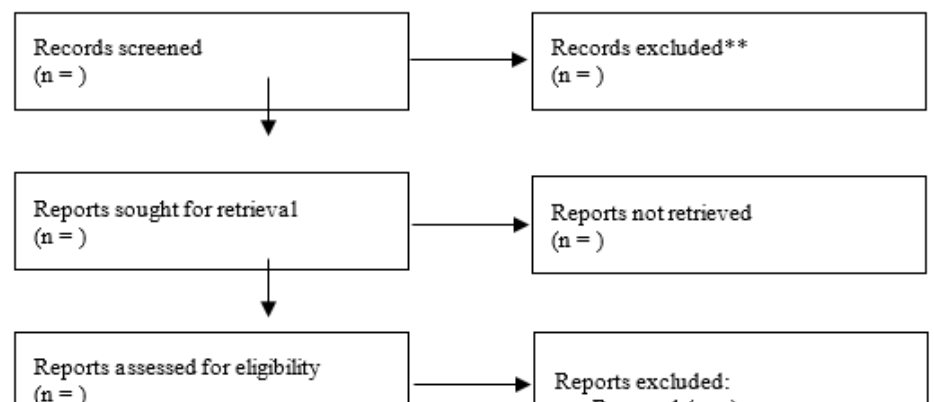

$(\mathrm{n}=)$

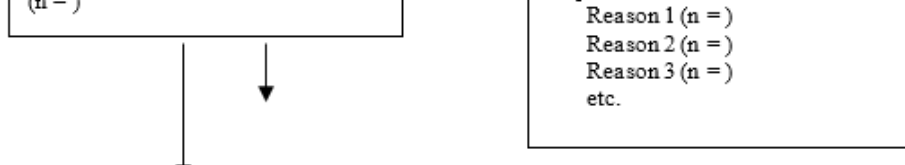

Studies included in review

$(\mathrm{n}=)$

Reports of included studies

$(\mathrm{n}=)$
Records identified from:

Websites $(\mathrm{n}=)$

Organisations ( $\mathrm{n}=$ )

Citation searching $(\mathrm{n}=)$

automation tools $(\mathrm{n}=)$

Records removed for other

etc.

Reports not retrieved

Reports sought for retrieval $(\mathrm{n}=)$

$(\mathrm{n}=)$

Reports excluded:

Reason $1(\mathrm{n}=)$

Reason 2( $\mathrm{n}=$ )

Reason $3(\mathrm{n}=)$

etc.

Figure 1

PRISMA 2020 flow diagram of the literature search

\section{Supplementary Files}

This is a list of supplementary files associated with this preprint. Click to download.

- S1PubMedSearchStrategy.docx

- S2PRISMAP2015Checklist.docx 